Proyecciones Journal of Mathematics

Vol. 31, No 2, pp. 165-168, June 2012.

Universidad Católica del Norte

Antofagasta - Chile

\title{
Hochschild-Serre Statement for the total cohomology
}

\author{
FRANÇOIS LESCURE \\ UNIVERSITÉ DE LILLE 1, FRANCE \\ Received : February 2012. Accepted : March 2012
}

\begin{abstract}
Let $M$ be a complex manifold and $\mathcal{F}$ a $O_{M}$-module with a $\mathbf{g}$ holomorphic action where $\mathbf{g}$ is a complex Lie algebra (cf. [3]). We denote by $\mathbf{H}(\mathbf{g}, \mathcal{F})$ the "total cohomology" as defined in [1] [2]. Then we prove that, for any ideal $\mathbf{a} \subset \mathbf{g}$, the module $\mathbf{H}^{\bullet}(\mathbf{a}, \mathcal{F})$ viewed as a $\mathrm{g} / \mathbf{a}$-module, we have a spectral sequence which converges to $\mathbf{H}(\mathbf{g}, \mathcal{F})$ and whose $E_{2}$-term is $E_{2}^{p, q}=H^{p}\left(\mathbf{g} / \mathbf{a} ; \mathbf{H}^{\mathbf{q}}(\mathbf{a}, \mathcal{F})\right)$.
\end{abstract}


Let $\mathbf{g}$ be a finite dimensional complex Lie algebra and $M$ a complex analytic manifold of finite dimension. Suppose that a holomorphic field $\mathbf{u}_{\mathbf{M}}$ of tangents $(1,0)$-vectors on $M$ is associated to each $\mathbf{u} \in \mathbf{g}$. If this transformation satisfies the condition $\left[\mathbf{u}_{\mathbf{M}}, \mathbf{v}_{\mathbf{M}}\right]=[\mathbf{u}, \mathbf{v}]_{\mathbf{M}}$, we shall say that it defines a holomorphic $\mathbf{g}$-action on $M$. To be more precise, the real parts of these fields $\mathbf{u}_{\mathbf{M}}$ are the opposite of the Killing fields of a local holomorphic action of some complex Lie group. Let $\mathcal{F}$ be an $O_{M}$-module and, for all $\mathbf{u} \in \mathbf{g}$, let $\gamma_{*}(\mathbf{u}): \mathcal{F} \rightarrow \mathcal{F}$ be a morphism of $C$-sheaf.

Definition 0.1. If, for any local section $\sigma$ of $\mathcal{F}$ and any local holomorphic function $f$ on $M$, we have:

(i) $\gamma_{*}([\mathbf{u}, \mathbf{v}])=\left[\gamma_{*}(\mathbf{u}), \gamma_{*}(\mathbf{v})\right]$

(ii) $\gamma_{*}(\mathbf{u})(\mathbf{f} \sigma)=\mathbf{L}_{\mathbf{u}_{\mathbf{M}}} \mathbf{f} \sigma+\mathbf{f} \gamma_{*}(\mathbf{u}) \sigma$, we say that $\mathcal{F}$ is an $O_{M}$-module with a holomorphic g-action.

Now, denote by $U(\mathbf{g}, \mathbf{C})$ be the envelopping algebra of the complex Lie algebra $\mathbf{g}$.

In [3], we have introduced the sheaf of crossed algebras $U\left(\mathbf{g}, \mathbf{O}_{\mathbf{M}}\right) \stackrel{\text { def }}{=}$ $\mathbf{O}_{\mathbf{M}} \otimes_{\mathbf{C}} \mathbf{U}(\mathbf{g}, \mathbf{C})$ with the use of the commutation formula: $(1 \otimes \mathbf{u})(\varphi \otimes \mathbf{1}) \stackrel{\text { def }}{=}$ $\mathbf{L}_{\mathbf{u}_{\mathbf{M}}} \varphi \otimes \mathbf{1}+\varphi \otimes \mathbf{u}$. Then, we see immediately that the $O_{M}$-modules with a holomorphic $\mathbf{g}$-action, are exactly the $U\left(\mathbf{g}, \mathbf{O}_{\mathbf{M}}\right)$-modules, objects which make some Abelian category denoted $\operatorname{Mod}\left(U\left(\mathbf{g}, \mathbf{O}_{\mathbf{M}}\right)\right)$. On the other hand, in [1] and [2], we have defined, for any holomorphically $G$-equivariant vector bundle $E \rightarrow M$ ( $G$ is a complex Lie group with Lie algebra $\mathbf{g}$ ), the total cohomology denoted $\mathbf{H}^{*}(\mathbf{g}, \mathbf{E})$. In [3], we have generalized this total cohomology to any $U\left(\mathbf{g}, \mathbf{O}_{\mathbf{M}}\right)$-module $\mathcal{F}$ and we have showed indeed that the total cohomology is a derived functor; more precisely, we have proved that:

$$
\mathbf{H}^{*}(\mathbf{g}, \mathbf{E}) \approx \mathbf{E x t}_{\mathbf{U}\left(\mathbf{g}, \mathbf{O}_{\mathbf{M}}\right)}^{*}\left(\mathbf{O}_{\mathbf{M}}, \mathbf{E}\right)
$$

Proposition 0.2. Let $M, \mathbf{g}$, and so on... be like above. Let $\mathcal{F}$ be a left $U\left(\mathbf{g}, \mathbf{O}_{\mathbf{M}}\right)$-module and $\mathbf{a}$ an ideal of the complex Lie algebra $\mathbf{g}$. Then:

(i) The total cohomology $\mathbf{H}(\mathbf{a}, \mathcal{F})$ is naturally a left $(\mathbf{g} / \mathbf{a})$-module.

(ii) There is a Hochschild-Serre spectral sequence $E_{r}$ whose $E_{2}$-term is given by $H^{p}\left(\mathbf{g} / \mathbf{a}, \mathbf{H}^{\mathbf{q}}(\mathbf{a}, \mathcal{F})\right)$ and which converges to $\mathbf{H}^{\mathbf{p}+\mathbf{q}}(\mathbf{g}, \mathcal{F})$

Proof. (i) It is well known, by the Poincaré-Birkhoff-Witt formula, that $U\left(\mathbf{g}, \mathbf{O}_{\mathbf{M}}\right)$ is a free left $U\left(\mathbf{a}, O_{M}\right)$-module, and then also, by the antiisomorphism $T$ (see [3]), a free right $U\left(\mathbf{a}, O_{M}\right)$-module. From this we deduce the exactness of the change of rings functor: 


$$
U\left(\mathbf{g}, \mathbf{O}_{\mathbf{M}}\right) \otimes \mathbf{U}\left(\mathbf{a}, \mathbf{O}_{\mathbf{M}}\right)-: \operatorname{Mod}\left(\mathbf{U}\left(\mathbf{a}, \mathbf{O}_{\mathbf{M}}\right)\right) \rightarrow \operatorname{Mod}\left(\mathbf{U}\left(\mathbf{g}, \mathbf{O}_{\mathbf{M}}\right)\right)
$$

By functor adjunction (see [3]), this exactness allows us to show that the 'forget functor': $\operatorname{Mod}\left(U\left(\mathbf{g}, \mathbf{O}_{\mathbf{M}}\right)\right) \rightarrow \operatorname{Mod}\left(\mathbf{U}\left(\mathbf{a}, \mathbf{O}_{\mathbf{M}}\right)\right)$ preserves injective objects. Also, taking the cohomology of the complex of global $\mathbf{a}$ - invariant sections of an injective resolution for an $U\left(\mathbf{g}, \mathbf{O}_{\mathbf{M}}\right)$-module $\mathcal{F}$, we obtain the total cohomology $\mathbf{H}^{\bullet}(\mathbf{a}, \mathcal{F})$ which is then a $(\mathbf{g} / \mathbf{a})$-module and does not depend of the auxiliary choice of the resolution.

(ii) The Grothendieck composition theorem of functors shows that it is sufficient to prove that, if $\mathcal{I}$ is an injective $U\left(\mathbf{g}, \mathbf{O}_{\mathbf{M}}\right)$-module, then the Chevalley-Eilenberg cohomology $H^{p}\left(\mathbf{g} / \mathbf{a}, \mathbf{H}^{\mathbf{0}}(\mathbf{a}, \mathcal{I})\right)$ of the $(\mathbf{g} / \mathbf{a})$-module $\mathbf{H}^{\mathbf{0}}(\mathbf{a}, \mathcal{I})$ is zero for $p \geq 1$. For this, we know that it will be enough - and we shall make it - to show that the $\mathbf{H}^{\mathbf{0}}(\mathbf{a}, \mathcal{I})$ is an injective $(\mathbf{g} / \mathbf{a})$-module.

Indeed, let $0 \rightarrow \mathbf{M}^{\prime} \stackrel{\mathbf{j}}{\rightarrow} \mathbf{M}$ be a monomorphism of $U(\mathbf{g} / \mathbf{a}, \mathbf{C})$-module. We must factorize each $(\mathbf{g} / \mathbf{a})$-morphism $\mathbf{M}^{\prime} \stackrel{\mathbf{u}}{\rightarrow} \mathbf{H}^{\mathbf{0}}(\mathbf{a}, \mathcal{I})$ through the monomorphism $\mathbf{j}$. Let us consider $\mathbf{M}^{\prime}$ and $\mathbf{M}$ as $\mathbf{g}$-modules with an ineffectiveness a; we introduce, as in [3], the $U\left(\mathbf{g}, \mathbf{O}_{\mathbf{M}}\right)$-modules $O_{M} \otimes_{C} \mathbf{M}^{\prime}$ and $O_{M} \otimes_{C} \mathbf{M}$, defined by the formula:

$$
\gamma_{*}(\mathbf{u})(\mathbf{f} \otimes \mathbf{m})=\mathbf{L}_{\mathbf{u}_{\mathbf{M}}} \mathbf{f} \otimes \mathbf{m}+\mathbf{f} \otimes \gamma_{*}(\mathbf{u}) \mathbf{m} .
$$

But, $\mathbf{j}$ enlarges it naturally in an arrow of $U\left(\mathbf{g}, \mathbf{O}_{\mathbf{M}}\right)$-modules $j: O_{M} \otimes_{C} \mathbf{M}^{\prime} \rightarrow$ $\mathbf{O}_{\mathbf{M}} \otimes_{\mathbf{C}} \mathbf{M}$. In more, $u$ allows to define naturally some arrow $O_{M} \otimes_{C} \mathbf{M}^{\prime} \rightarrow \mathcal{I}$ which, by the injectivity of $\mathcal{I}$, factorizes itself by $\mathbf{j}$ with the use of one arrow: $O_{M} \otimes_{C} \mathbf{M} \rightarrow \mathcal{I}$.

Last arrow that defines one other: $\mathbf{H}^{\mathbf{0}}\left(\mathbf{a}, \mathbf{O}_{\mathbf{M}} \otimes_{\mathbf{C}} \mathbf{M}\right) \rightarrow \mathbf{H}^{\mathbf{0}}(\mathbf{a}, \mathcal{I})$. But, then, by restriction of this last arrow to $\mathbf{M} \subset \mathbf{H}^{\mathbf{0}}\left(\mathbf{a}, \mathbf{O}_{\mathbf{M}} \otimes_{\mathbf{C}} \mathbf{M}\right)$, we see easily that this answers the question. 


\section{References}

[1] F. Lescure, Action sur la cohomologie de Dolbeault, C. R. Acad. Sci., 314, pp. 923-926, (1992).

[2] F. Lescure, Cohomologie totale et courants de Dolbeault invariants, J. reine angew. Math., 475, pp. 103-136, (1996).

[3] F. Lescure La cohomologie totale est un foncteur dérivé, Homology, Homotopy and Applications, Volume 12, Number 1, pp. 367-400, (2010).

François Lescure

U.M.R. CNRS 8524

U.F.R. de Mathématiques

Université de Lille 1

59655 Villeneuve d'Ascq Cedex

FRANCE

e-mail : francois.lescure@math.univ-lille1.fr 